\title{
An Optimized Biological Taser: Electric Eels Remotely Induce or Arrest Movement in Nearby Prey
}

\author{
Kenneth C. Catania \\ Department of Biological Sciences, Vanderbilt University, Nashville, Tenn., USA
}

\section{Key Words}

Efferents · Electric fish · Gymnotidae · Muscle · Predator .

Prey $\cdot$ Strongly electric species $\cdot$ Taser

\begin{abstract}
Despite centuries of interest in electric eels, few studies have investigated the mechanism of the eel's attack. Here, I review and extend recent findings that show eel electric high-voltage discharges activate prey motor neuron efferents. This mechanism allows electric eels to remotely control their targets using two different strategies. When nearby prey have been detected, eels emit a high-voltage volley that causes whole-body tetanus in the target, freezing all voluntary movement and allowing the eel to capture the prey with a suction feeding strike. When hunting for cryptic prey, eels emit doublets and triplets, inducing whole-body twitch in prey, which in turn elicits an immediate eel attack with a full volley and suction feeding strike. Thus, by using their modified muscles (electrocytes) as amplifiers of their own motor efferents, eel's motor neurons remotely activate prey motor neurons to cause movement (twitch and escape) or immobilization (tetanus) facilitating prey detection and capture, respectively. These results explain reports that human movement is 'frozen' by eel discharges and shows the mechanism to resemble a law-enforcement Taser.

(c) 2015 The Author(s)

Published by S. Karger AG, Basel
\end{abstract}

\section{KARGER 125\%}

E-Mail karger@karger.com www.karger.com/bbe

This article is licensed under the Creative Commons Attribution-

\section{(9) 2015 The Author(s) \\ Published by S. Karger AG, Basel 0006-8977/15/0861-0038\$39.50/0} NonCommercial-NoDerivatives 4.0 International License (CC BYNC-ND) (http://www.karger.com/Services/OpenAccessLicense) Usage and distribution for commercial purposes as well as any distribution of modified material requires written permission.

\section{Introduction}

The electric eel (Electrophorus electricus) stands out as a remarkable product of evolution. It is the most powerful electrogenic animal, with most of its body composed of electrocytes providing a combined discharge that can exceed $600 \mathrm{~V}$ [Grundfest, 1957]. It is, therefore, not surprising that people have been aware of the special powers of these fish for centuries of recorded history, and no doubt for millennia of unrecorded history. Any South American fisherman who caught an electric eel would have an impressive story to tell. But the most famous tale has to be Alexander von Humboldt's account of a battle between horses and eels in 1800 [von Humboldt, 1807]. At the time, he was collecting eels in a quest to understand the nature of electricity, and the strategy of the local fishermen was to exhaust the eels by herding wild horses into the water. Some horses reportedly died during the encounter (perhaps from drowning), but von Humboldt got his eels and performed his experiments. In 1838, Faraday extended von Humboldt's experiments to observe an eelgenerated spark, confirming the electrical nature of the eel's discharge [Faraday, 1838]. Electric eels and other strongly electric fish were thus useful for our early understanding of electricity [Williamson and Walsh, 1775] and helped inspire Volta's invention of the battery, which he

Kenneth C. Catania

Department of Biological Sciences, Vanderbilt University

VU Station B, Box 35-1634

Nashville, TN 37235 (USA)

E-Mail ken.catania@vanderbilt.edu 


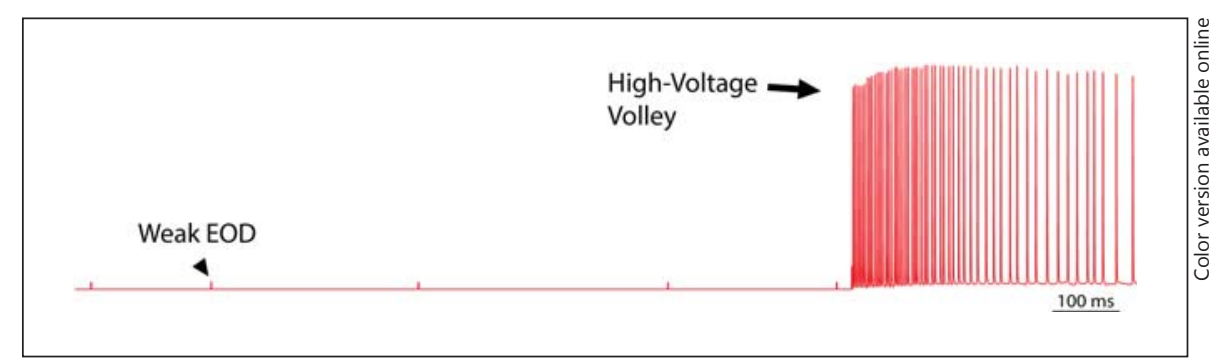

Fig. 1. Recording showing the 2 different EODs of an electric eel. Each is a monophasic, head-positive pulse lasting approximately $1 \mathrm{~ms}$. The low-voltage output (arrowhead) occurs at a low rate, usually less than $10 \mathrm{~Hz}$, and is used for electrolocation. The rate of this weak discharge increases dramatically when eels hunt or en- counter novel objects. The high-voltage output (arrow) is much stronger and occurs at a much higher rate in volleys of up to 500 $\mathrm{Hz}$ during the predatory strike. High-voltage volleys are also used for defense.
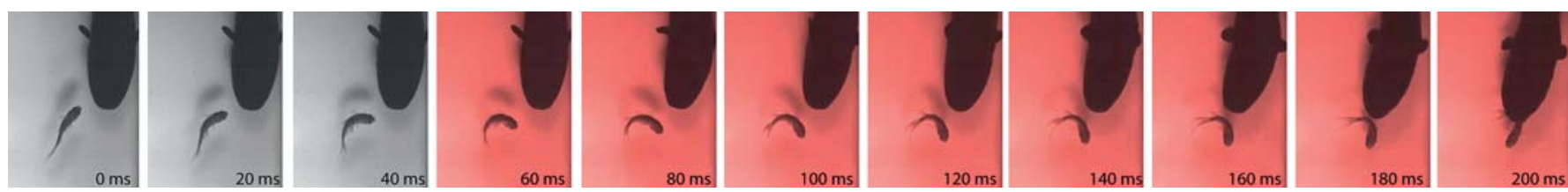

a
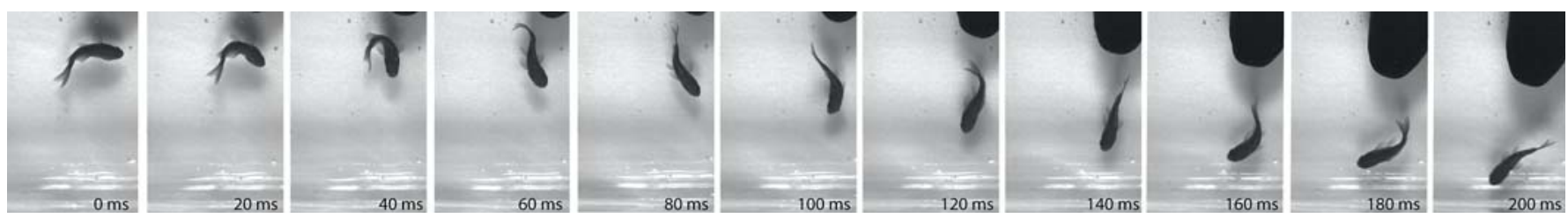

b

Fig. 2. Images captured from a high-speed video showing the movements of an eel and prey fish. a An approaching eel elicits a $\mathrm{C}$-start escape response in a goldfish, but the goldfish is immobilized by a volley of high-voltage pulses (red frames) and captured within $200 \mathrm{~ms}$. b A similar encounter during which the fish was not immobilized and thus rapidly outpaced the eel. In the final frame (far right), this distance between eel and fish has increased and the prey velocity is greater than the eel velocity. called an 'artificial electric organ' similar to the eel's [Volta, 1800]. In the 20th century, electric eels played a role in the isolation of the acetylcholine channel [Changeux et al., 1970; Keesey, 2005] and in determining the structure of voltage-gated sodium channels [Noda et al., 1983]. Most recently, the electric eel genome has been sequenced, providing insights into the basis of convergent evolution of electric organs [Gallant et al., 2104], a problem pondered by Darwin in On the Origin of Species [Darwin, 1873]. Yet, despite this storied history and the many contributions electric eels have made to science, few studies have investigated the details of how the eel's electric discharge affects prey. In a recent investigation, I showed that electric eel high-voltage volleys remotely activate the motor neurons of nearby animals, allowing them to rapidly incapacitate potential prey by inducing massive, involuntary muscle contraction [Catania, 2014]. Alternatively, using pairs or triplets of high-voltage pulses, eels can cause involuntary twitch in nearby animals, revealing their location to the eel. Here, I review and extend the evidence for these abilities, and discuss the implications of this mechanism for the evolution of eel discharge patterns.

\section{Electric Eels Emit Both Low- and High-Voltage Pulses}

Electric eels are unique among strongly electric fish in giving off two different electric organ discharges (EODs) of different intensity (fig. 1). As Bauer [1979] and others [Coates et al., 1940; Hagiwara et al., 1965; Bullock, 1969; 
Westby, 1988] have shown, a low-amplitude EOD is produced at low frequencies (typically $<10 \mathrm{~Hz}$ ) and consists of an approximately 2-ms monophasic, head-positive pulse that is part of the eel's electrosensory system [Keynes and Martins-Ferreira, 1953; Hagiwara et al., 1965]. The high-voltage EOD is also a monophasic, head-positive pulse of about 2-ms duration, but it is far more powerful and emitted at much higher frequencies during prey capture or defense. Volleys of the high-voltage EOD are always immediately preceded by a single low-voltage EOD [Bauer, 1979]. This review focuses on the high-voltage EOD.

\section{Eel High-Voltage Discharges Rapidly Freeze Prey Movement}

I became interested in the mechanistic details of the eel's strike after recording eel predatory behavior in very slow motion (at 1,000 frames/s). The recordings showed that eels arrest voluntary movement in nearby fish in roughly $3 \mathrm{~ms}$ using their high-voltage discharge (fig. 2a). The usefulness of this ability was clear when slow-motion videos of eels approaching immobilized and escaping fish were compared. Although eels combine their high-voltage discharge with a very rapid suction feeding strike, prey can outpace the eel unless they are immobilized (fig. 2b). Eel high-voltage volleys are often of short duration and fish will generally recover and continue to flee if not captured during the strike. Thus, the high-voltage volley provides the eel with a critical advantage for a brief period during which fish would otherwise make use of their rapid escape abilities to evade the strike (though prey electrified by close, large eels may be immobilized for longer periods following the high-voltage volley - see later section).

\section{Prey Are Immobilized by Induction of Massive Whole-Body Muscle Tension}

The speed with which eels inactivate prey was remarkable and the mechanism was not obvious from the slowmotion video. It seemed possible that eels were activating the fish neuromuscular system [Catania, 2014], as maximal contraction of opposing musculature during an escape (or in a stationary fish) could presumably freeze voluntary motion as equally powerful muscles contracted in opposition. This hypothesis was tested with a whole-fish muscle preparation in which a pithed fish was attached to a force transducer while a nearby eel was fed earthworms, which it shocked with its high-voltage discharge (fig. 3a). The high-voltage volleys induced strong muscle contraction in the nearby fish with a latency of $3.4 \mathrm{~ms}$. This closely matched the mean $(2.9 \mathrm{~ms})$ latency of immobilization observed in the high-speed video [Catania, 2014].

It was important to also determine whether the amount of muscle tension developed by the eel's volley was sufficient to incapacitate the fish. This was estimated by measuring the maximal tension that could be developed in the fish preparation by direct electric stimulation with leads attached to the output of a Grass SD9 stimulator (fig. 3b). Nearby eels consistently induced near maximal tension in prey fish [Catania, 2014]. These results indicated that eels immobilize their prey by inducing involuntary muscle contractions in the manner analogous to a law-enforcement Taser [Sweeney, 2009]. To avoid potential injuries, Tasers are engineered to induce muscle contractions in humans at a comparatively low tension (19 pulses/s). Nevertheless, this submaximal tension is sufficient to prevent movement in humans. Submaximal tension is also likely to disrupt fish movements and, if so, this may suggest how the eel's strong EOD evolved from ancestral species with weaker discharges. Presumably, even a short disruption or delay in the prey's C-start escape would provide eels with an advantage. Young, small eels could provide clues to the utility of less powerful EODs.

\section{Eels Induce Prey Muscle Tension by Activating Motor Neuron Efferents}

To determine whether eels activated prey muscles directly, or through motor neurons, the experiments described above were repeated with two fish stationed side by side, each attached to a different force transducer. One fish was curarized to block the neuromuscular junction, and the other was sham injected with saline. In each case, tension responses dropped to near zero in the curarized fish [Catania, 2014]. This experiment indicated that the eel's high-voltage volley does not directly activate muscles, but rather the muscle contractions require activation of the presynaptic components of the neuromuscular junction. To determine whether this activity required an intact central nervous system, the tension experiments were repeated in double-pithed fish, in which both the brain and the spinal cord had been destroyed, compared to brain-pithed fish. No difference was observed in the double-pithed fish compared to the brain-pithed fish. The conclusion from these experiments is that volleys of 


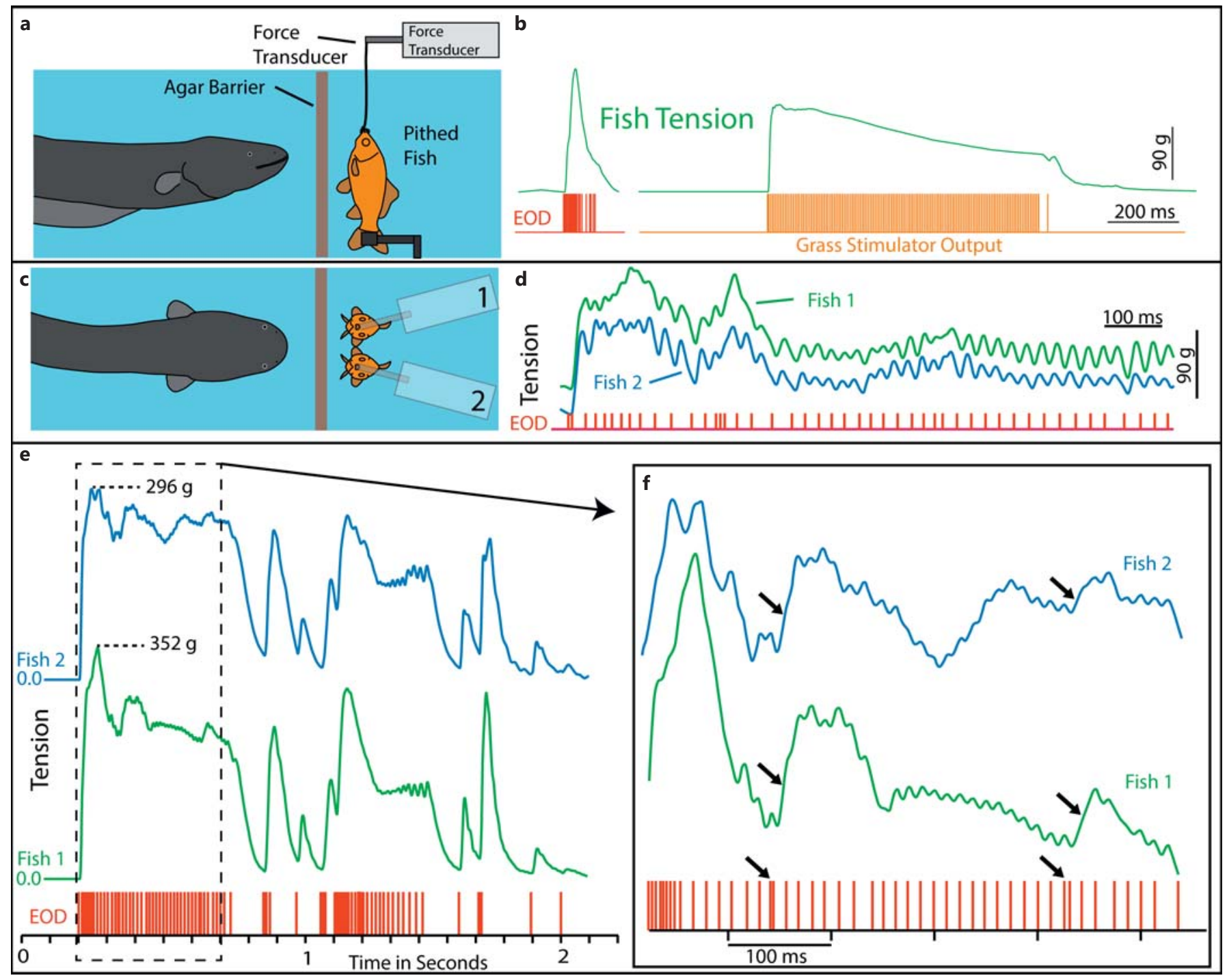

Fig. 3. Paradigm and results for eel-induced muscle tension measurements in prey. a To measure prey muscle tension, a pithed fish was attached to a force transducer while an eel (behind an agar barrier) was fed earthworm (which it shocked with high-voltage volleys). b Onset of fish tension (green) occurred in roughly $3 \mathrm{~ms}$ and was generally similar to the maximum whole-body fish tension that could be induced through direct stimulation (orange trace) with an SD9 Grass stimulator. c To compare responses of two different fish under various conditions [Catania, 2014], two force transducers were placed side by side. $\mathbf{d}$ The dual fish para-

the eel's high-voltage EOD activate efferents of motor neurons to induce muscle tension in nearby prey.

The dual tension experiments described above also revealed two unanticipated details about the consistency and fidelity of the eel's activation of prey motor neurons [Catania, 2014]. The first was that eels produced remark- digm unexpectedly revealed that long eel interpulse intervals result in nearly identical patterns (green and blue traces) of individual twitches in the two adjacent fish. e Overall tension responses in two fish were also similar at a more compressed time scale (blue and green traces, different cases from $\mathbf{d}$ ). f An expanded time scale shows the marked effect of eel doublets - closely spaced EODs (arrows) - on corresponding fish tension. This suggests that doublets have the same strong tension-inducing effect in fish as shown for other experimental preparations. 
As eels were fed earthworms repeatedly during tension experiments, the interpulse interval of their high-voltage discharge increased in some trials (likely as a result of fatigue). When this happened, individual whole-body twitches often emerged on both fish tension records, with each twitch corresponding to a single eel pulse (fig. 3d-f). This result indicates that each pulse of the eel's high-voltage discharge typically produces an action potential in the efferent branches of motor neurons in nearby animals. As would be expected for any muscle, high frequencies of action potentials in the motor neuron caused a fused muscle tetanus, whereas only at lower frequencies were individual twitches apparent. Of course, fish possess a myriad of different efferents and muscles with both fast (white) and slow (red) fiber types [Granzier et al., 1983]. It is possible that the peaks on the tension trace correspond primarily to twitches from the largest, fast fibers of the trunk, and that slower and smaller muscles lag this response but do not produce sufficient tension to appear independently on the trace. In any case, the tension results indicate that eels are remotely controlling the motor neurons of their targets with high fidelity. This observation suggests the eel's high-voltage volley should be considered from the prey's perspective. What are the implications of the temporal pattern of discharges in the context of prey motor neuron action potentials?

\section{Eel High-Voltage Discharges Resemble Optimal Motor Neuron Trains}

A long history of investigation of neuromuscular systems has revealed two particular features in the motor neuron train that maximize the rate of muscle tension development. These are (1) a short interpulse interval for the first two motor neuron action potentials (a doublet) in the stimulating train [Burke et al., 1976; Zajac and Young, 1980a, b; Hennig and Lømo, 1985; Celichowski and Grottel, 1998; Thomas et al., 1999; Binder-Macleod and Kesar, 2005; Cheng et al., 2013; Pedersen et al., 2013] and (2) high frequency of the motor neuron train [Buller and Lewis, 1965; Rack and Westbury, 1969; Fuchs and Luschei, 1971; Lewis and Proske, 1972; De Haan, 1998]. The eel's highvoltage volley exhibits both of these features.

Analysis of the first 11 impulses (the first 10 interpulse intervals) from volleys from each of 4 different electric eels showed that each begins with 2 pulses that have a significantly shorter interpulse interval than the remaining 9 interpulse intervals (fig. 4). In addition to this initial doublet, the roughly $300-$ to $500-\mathrm{Hz}$ frequency of the

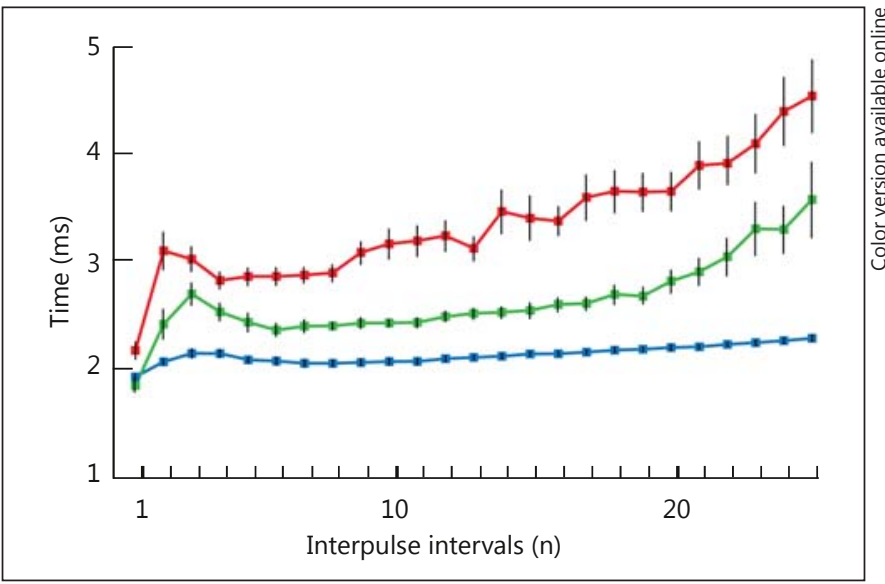

Fig. 4. The mean interpulse intervals for differently sized electric eels. The longest interpulse intervals (red) corresponded to the smallest eel $(50 \mathrm{~cm})$, whereas the shortest intervals (blue) corresponded to the largest eel $(115 \mathrm{~cm})$. For each specimen, the first interpulse interval was the shortest. Bars are standard errors. (See Catania [2014] for additional statistics.) The colors refer to the online version only.

EOD has been shown (for motor neuron stimulation) to correspond to high rates of tension development in experimental preparations. Moreover, the overall distribution of pulses in the eel's high-voltage volley resembles motor neuron trains found to be near optimal for muscle tension development [Zajac and Young, 1980a, b]. Such trains often begin with a doublet, followed by a brief postdoublet decrease in frequency, and then continuation of the motor neuron action potential train [Zajac and Young, 1980b]. This is the pattern observed for eel volleys, although the eel's EOD has a much higher overall frequency than that typical for motor neurons.

These comparisons suggest that the temporal pattern of eel high-voltage volleys may have been selected to most efficiently induce very rapid and powerful muscle contractions and, hence, immobilization of nearby prey. One potential objection to this conclusion might be that such temporal patterns (a doublet followed by very high frequencies of action potentials) are not typically observed from vertebrate motor neurons. Put another way, if eels are so good at generating rapid and massive (supernormal) tension in prey with their high-voltage discharge patterns, why have not identical (though shorter) temporal patterns of action potentials been adopted by the target animals to produce their own, supernormal, rapid muscle contraction during normal behavior?

Such high-frequency patterns of muscle activation cannot be used, even briefly, for voluntary movements

\section{Catania}


because muscles in behaving animals must very rapidly uncontract. As pointed out by Rome [2006] and other investigators [Zajac and Young, 1980b; Rome et al., 1996, 1999; Harwood et al., 2011], a trade-off exists between tension development and subsequent muscle use. Although higher rates of muscle tension development than are typically observed in vivo may theoretically (or experimentally) be evoked and maintained with higher motor neuron discharge rates, subsequent fatigue or inability to resorb calcium and relax make this an untenable strategy for muscles producing body movements in behaving animals. Zajac and Young [1980b] suggest that optimal patterns of normal motor activity are constrained in number of discharges (and hence constrained to produce submaximal tension) to keep neuromuscular and muscle fiber fatigue at an acceptable level. From the eel's perspective, this constraint is an opportunity. The overactivation of prey motor neurons causes rapid development of tension while presumably overwhelming the muscle's calcium pumps [Rome, 2006], intensifying and prolonging the immobilizing tension that prevents prey escape. This may explain why, in some cases, fish remain immobilized for several seconds after the eel's high-voltage volley. Their muscles may simply be completely fatigued following the high frequency of action potentials induced by the eel's discharge.

In addition to these considerations, the teleost fish neuromuscular junction is poly-innervated and less secure than its mammalian counterpart, and fish red muscle may have a graded response to excitation [Johnston, 1980, 1982; Granzier et al., 1983; Kamata and Washio, 2003]. Thus induction of spatial and temporal summation at the fish muscle may play a role in the eel's attack, particularly when fish are at longer distances where individual pulses of the EOD may produce subthreshold potentials in prey efferents. Finally, facilitation at the prey's presynaptic terminal may occur during the eel's high-frequency discharge to further activate the muscles [Mallart and Martin, 1967]. All of these considerations suggest that the form of the eel's EOD is very well suited to efficiently induce rapid and powerful involuntary muscle contraction in nearby animals.

\section{Eels Induce Movement in Cryptic Prey with Isolated Doublets and Triplets}

As briefly described above, a doublet at the outset of a motor neuron train produces rapid onset of strong muscle tension. The effect of such closely spaced action potentials on muscle tension is generally studied in an isolated nervemuscle preparation using a calibrated electrical stimulator at a frequency set by the experimenter. Remarkably, evidence for the effect of a doublet on muscle tension relative to more widely spaced action potentials can be found in the responses of fish to the eel's high-voltage discharge. Figure $3 \mathrm{f}$ shows an expanded view from the dual tension experiments with the pulse train (red) below the tension responses of the two fish (green and blue). In this example, the eel's high-voltage volley has unusually long and variable interpulse intervals because the eel was fatigued from prolonged feeding on earthworms. Individual twitches are evident for each fish in response to the EOD. In two places (arrows), the eel's interpulse interval was short (a doublet). In response to both doublets, the tension in each fish was substantially elevated (arrows). Thus, fish muscles seem to respond to eel doublets as would be predicted from investigation of other neuromuscular systems in more controlled settings.

Given these findings, it was of particular interest that hunting eels give off periodic, isolated high-voltage doublets (and triplets) as they search for prey. This had been previously noted [Bauer, 1979] and was observed for all of the eels in the most recent study [Catania, 2014]. This behavior also occurred many times during the fish tension experiments, and, in every case, the doublet induced a strong twitch response in the pithed fish (fig. 5a). Although the fish tension experiments were designed to investigate the reaction of prey muscles to eel high-voltage volleys, these same experiments fortuitously provided unanticipated information about eel hunting behavior. This happened when eels approached the agar barrier in front of the pithed fish (fig. 3a) and gave off a doublet. On several occasions, the doublet was followed by an immediate and violent attempt to break through the barrier to reach the fish preparation (the barrier invariably had to be replaced when this happened). The eels had clearly transitioned from an exploratory phase to an attack phase following the doublet. The doublet somehow informed the eel about the nearby prey. It is important to note in this regard that the thin agar barrier (reinforced with nylon netting) used in the tension studies was for minimizing diffusion of residual anesthesia or curare from the pithed fish preparation and did not mask mechanosensory cues. These observations, together with the appreciation that doublets in motor neuron trains are optimal for maximizing muscle response, suggested that isolated doublets and triplets could function by generating involuntary movements in cryptic prey that eels detect from mechanosensory and/or electrosensory cues. 


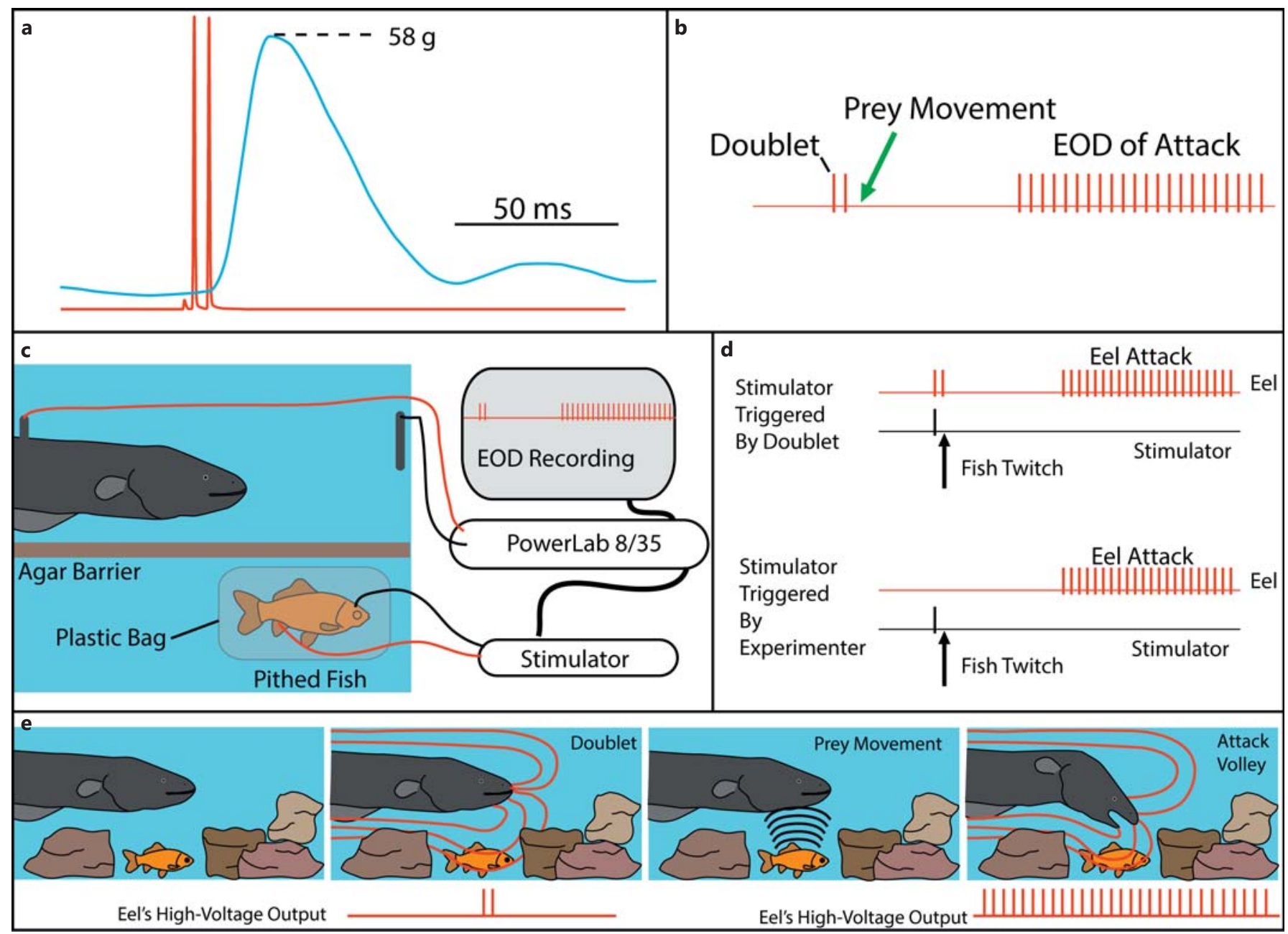

Fig. 5. The use of doublets in eel predatory behavior. a Example of an isolated doublet (red) inducing strong tension (blue) in a nearby pithed fish attached to a force transducer. $\mathbf{b}$ Schematic of the doublet output followed by prey movement (twitch) and then a full high-voltage volley. c Schematic of the paradigm used to investigate the use of doublets during hunting. A pithed fish was enclosed in a plastic bag, while connected to an SD9 Grass stimulator that

To explore this possibility further, live prey (fish, crayfish and tadpoles) were presented to hunting eels below an agar barrier [Catania, 2014]. Often, the eel seemed to track and detect the prey using its low-voltage EOD (which was given off at maximal frequency during these encounters) and then attacked directly with a high-voltage volley and suction feeding strike. But, on other occasions, the eel gave off a doublet while investigating the agar barrier. Doublets always induced movement in all prey types, and were then quickly followed by a full, immobilizing volley of high-voltage pulses and a suction feeding strike. could induce twitch when the eel emitted a doublet. $\mathbf{d}$ The doublet was followed by a full volley (and predatory strike) if twitch was immediately triggered through the stimulator (upper trace), but no attacks were elicited in the absence of prey twitch (not shown). In the absence of doublets, full volleys and strikes could be elicited by randomly generated fish twitch (bottom). e Schematic illustration of the use of doublets to detect prey in normal hunting behavior.

The form of the entire EOD trace in these trials was distinct from previously analyzed attacks on free-swimming prey. It consisted of a doublet (or triplet) followed by a 20 - to $40-\mathrm{ms}$ pause (during which prey moved) and then a full EOD volley (fig. 5b). Such lengthy pauses in attack volleys were never seen during attacks on freeswimming prey or in response to mechanosensory stimulation (water pulses). In addition, details of the waveform for isolated doublets and triplets were generally unique and eel specific. As a result, doublets and triplets usually had the same distinctive waveform whether in isolation or when followed by an attack volley. These observations 
further strengthened the hypothesis that eels make use of doublets and triplets to detect cryptic prey by inducing movement.

To investigate this hypothesis in detail, a series of experiments was designed to determine how eels responded to doublet-induced electrical and mechanosensory stimuli. No responses were obtained from doublet-induced artificial electrical stimuli in the form of microvolt and millivolt dipole fields (not shown). However, the form of electric field generated by a fish whole-body twitch is not obvious nor is it easily simulated. Therefore, negative results from preliminary experiments using electric stimuli remain inconclusive.

It was, however, possible to exactly produce the mechanosensory cues caused by a fish in response to an eel doublet. This was accomplished by placing a pithed fish in a thin plastic bag under an agar barrier, with electrical leads embedded in the head and tail region that could independently trigger fish twitch from the PowerLab module through an SD9 Grass stimulator (fig. 5c). This allowed precise experimental control over the timing and occurrence of the mechanosensory stimulus (fish twitch) usually generated by the eel's doublet (hydrophone recordings confirm that fish twitches generate hydrodynamic stimuli that pass through the thin plastic bag and agar barrier).

In the absence of an SD9-stimulator-induced twitch, eel doublets while exploring the preparation caused no pithed-fish response (the plastic bag electrically isolated the fish) and were never followed by full attack volley or strikes. But when the stimulator triggered fish twitch following an eel's doublet, the eel follows up with a full volley of high-voltage pulses and a suction feeding strike. The eel's behavior in this paradigm was identical to the natural 'doublet attack' observed for intact prey.

To investigate whether the latency from doublet to full volley (20-40 ms) was consistent with the eel's reaction time for producing an EOD in the absence of a doublet, the pithed fish was occasionally induced to twitch by triggering the stimulator independent of the eel's doublet (fig. 5d). Eels attacked with a full volley and suction feeding strike with the same latency as observed for doublet-triggered twitch. The remarkably short attack latency $(20-40 \mathrm{~ms})$ results in eel doublet attacks that appear virtually instantaneous to the naked eye. It is only by reviewing a slow motion video or examining the eel's volley at high time resolution that most doublets followed by attacks became evident.

A range of control experiments confirmed that eels were responding to twitch-generated mechanosensory cues in the above paradigm and that doublets were not

Electric Eels Remotely Induce or Arrest Movement followed by attack in the absence of fish movement. These include trials with the doublet-triggered electrical leads in the bag but no pithed fish, trials with the doublet-triggered leads attached to a nonviable fish preparation (frozen and thawed pithed fish), trials with the viable pithed fish but stimulator inactive, trials with a freeze-thawed fish but no plastic bag, and trials with the agar barrier replaced with Plexiglas to control for visual-motion cues and other variations [Catania, 2014].

In addition to revealing a role for doublets in eel hunting behavior, these experiments and related behavioral observations showed that eels are exquisitely sensitive to prey-generated mechanosensory cues and are primed to attack the source of water movement. The typical response to such stimuli is an immediate high-voltage discharge and strike. These observations suggest that some incidents of human or cattle electrification by eels may be the result of misplaced eel predatory attacks, rather than defensive behaviors.

\section{Conclusions}

The results of recent investigations show that highvoltage discharges of electric eels remotely activate motor neurons in nearby animals. Using this ability, electric eels can either immobilize or activate prey depending on the situation. The immobilization mechanism described here, which is the same as a law-enforcement Taser, explains historical accounts of people being frozen in place by electric eels. In the late 1800 s, Sachs reported that locals 'attempting to get out of a boat into the water are frequently unable to either get in or out after touching the water if an eel is near by, until shock ceases' [Ellis, 1913; Finger and Piccolino, 2011]. Sachs himself dropped an eel on his legs and while shocked was unable to move for $30 \mathrm{~s}$ [Ellis, 1913]. Alexander von Humboldt reported that the Tamarac Indians of South America called electric eels 'arimna' which means 'something that deprives of motion' [Ellis, 1913]. These accounts, together with the present results, indicate that electric eels can immobilize a wide range of vertebrates.

Electric eels have also leveraged the remote-control mechanism to cause involuntary movement in nearby prey. This is an astounding adaptation that hijacks the prey's own nervous system to cause a massive whole-body twitch. The induced movement is probably larger than could be produced voluntarily, because all of the animal's musculature may be involved. This in turn produces a mechanosensory cue (water movement) ideal for a hunting eel. 
Other strongly electric species may use the same mechanisms. Strongly electric rays in the family Torpedinidae derive their name not from the military weapon (the reverse is true) but rather from the term torporific - depriving of motion. Belbenoit and Bauer [1972] suggest the EOD of Torpedo marmorata may also serve to startle prey. In the case of electric catfish, Belbenoit et al. [1979] recorded the EODs of hunting strongly electric catfish (Malapterurus electricus) and report the occurrence of short 'pre-volleys'. They proposed catfish might use these pre-volleys to startle immobile prey in order to detect them through mechanosensation. If so, this would be further evidence for the independent convergence of electrical hunting strategies across species.

But the most impressive and prescient deduction about the electric eel hunting strategy and behavior comes not from a biologist, but from the towering historical figure and father of electromagnetism, Michael Faraday. In 1838 , when our understanding of nervous systems and muscles was in its infancy, Michael Faraday experimented with electric eels. After using a literally 'hands on' approach, he concluded [Faraday, 1838]:
The Gymnotus appears to be sensible when he has shocked an animal, being made conscious of it, probably, by the mechanical impulse he receives, caused by the spasms into which it is thrown. When I touched him with my hands, he gave me shock after shock; but when I touched him with glass rods, or the insulated conductors, he gave one or two shocks, felt by others having their hands in at a distance, but then ceased to exert influence, as if made aware it had not the desired effect. Again, when he has been touched with the conductors several times for experiments on the galvanometer or other apparatus, and appears to be languid or indifferent, and not willing to give shocks, yet being touched by the hands, they, by convulsive motion, have informed him that a sensitive thing was present, and he has quickly shown his power and willingness to astonish the experimenter.

\section{Acknowledgments}

This work was supported by NSF grant 1456472 to K.C.C. I thank Elizabeth Catania for comments on the paper and advice on experimental approaches. All procedures were approved by the Vanderbilt Institutional Animal Care and Use Committee.

\section{References}

Bauer R (1979): Electric organ discharge (EOD) and prey capture behaviour in the electric eel, Electrophorus electricus. Behav Ecol Sociobiol 4:311-319.

Belbenoit P, Bauer R (1972): Video recordings of prey capture behaviour and associated electric organ discharge of Torpedo marmorata (Chondrichthyes). Marine Biol 17:93-99.

Belbenoit P, et al. (1979): Ethological observations on the electric organ discharge behaviour of the electric catfish, Malapterurus electricus (Pisces). Behav Ecol Sociobiol 4:321330 .

Binder-Macleod S, Kesar T (2005): Catchlike property of skeletal muscle: recent findings and clinical implications. Muscle Nerve 31: 681-693.

Bullock TH (1969): Species differences in effect of electroreceptor input on electric organ pacemakers and other aspects of behavior in electric fish. Brain Behav Evol 2:85-101.

Buller AJ, Lewis DM (1965): The rate of tension development in isometric tetanic contractions of mammalian fast and slow skeletal muscle. J Physiol 176:337-354.

Burke RE, Rudomin P, Zajac FE III (1976): The effect of activation history on tension production by individual muscle units. Brain Res 109:515-529.
Catania K (2014): The shocking predatory strike of the electric eel. Science 346:1231-1234.

Celichowski J, Grottel K (1998): The influence of a doublet of stimuli at the beginning of the tetanus on its time course. Acta Neurobiol Exp (Wars) 58:47-54.

Changeux J-P, Kasai M, Lee CY (1970): Use of a snake venom toxin to characterize the cholinergic receptor protein. Proc Natl Acad Sci USA 67:1241-1247.

Cheng AJ, Place N, Bruton JD, Holmberg HC Westerblad H (2013): Doublet discharge stimulation increases sarcoplasmic reticulum $\mathrm{Ca}^{2+}$ release and improves performance during fatiguing contractions in mouse muscle fibres. J Physiol 591:3739-3748.

Coates CW, Cox RT, Rosemblith WA, Brown MB (1940): Propagation of the electric impulse along the organs of the electric eel, Electrophorus electricus (Linnaeus). Zoologica 25: 249-256.

Darwin C (1873): The Origin of Species by Means of Natural Selection, or the Preservation of Favoured Races in the Struggle for Life, ed 6. London, Murray.

De Haan A (1998): The influence of stimulation frequency on force-velocity characteristics of in situ rat medial gastrocnemius muscle. Exp Physiol 83:77-84.
Ellis MM (1913): The Gymnotid Eels of Tropical America (No. 77). Pittsburgh, Carnegie Institute.

Faraday M (1838): Experimental researches in electricity. Philos Trans R Soc Lond 122:125162

Finger S, Piccolino M (2011): The Shocking History of Electric Fishes: From Ancient Epochs to the Birth of Modern Neurophysiology. Oxford, Oxford University Press, p 5.

Fuchs AF, Luschei ES (1971): Development of isometric tension in simian extraocular muscle. J Physiol 219:155.

Gallant JR, Traeger LL, Volkening JD, Moffett H, Chen PH, Novina CD, Sussman MR (2014): Genomic basis for the convergent evolution of electric organs. Science 344:1522-1525.

-Granzier HLM, Wiersma J, Akster HA, Osse JWM (1983): Contractile properties of a white- and a red-fibre type of the m. hyohyoideus of the carp (Cyprinus carpio L.). J Comp Physiol 149:441-449.

Grundfest H (1957): The mechanisms of discharge of the electric organs in relation to general and comparative electrophysiology. Prog Biophys Biophys Chem 7:1-85.

Hagiwara S, Szabo T, Enger PS (1965): Physiological properties of electroreceptors in the electric eel, Electrophorus electricus. J Neurophysiol 28:775-783. 
Harwood CL, Young IS, Tikunov BA, Hollingworth S, Baylor SM, Rome LC (2011): Paying the piper: the cost of $\mathrm{Ca}^{2+}$ pumping during the mating call of toadfish. J Physiol 589:54675484.

Hennig R, Lømo T (1985): Firing patterns of motor units in normal rats. Nature 314:164-166.

Johnston IA (1980): Contractile properties of fish fast muscle fibres. Mar Biol Lett 1:323-328.

Johnston IA (1982): Biochemistry of myosins and contractile properties of fish skeletal muscle. Mol Physiol 2:15-29.

Kamata R, Washio H (2003): Neuromuscular transmission of pectoral fin muscles of the goldfish Carassius auratus. Comp Biochem Physiol A Mol Integr Physiol 135:435-442.

Keesey J (2005): How electric fish became sources of acetylcholine receptor. J Hist Neurosci 14: 149-164.

Keynes RD, Martins-Ferreira H (1953): Membrane potentials in the electroplates of the electric eel. J Physiol 119:315-351.

Lewis DM, Proske U (1972): The effect of muscle length and rate of fusimotor stimulation on the frequency of discharge in primary endings from muscle spindles in the cat. J Physiol 222: 511-535.

Mallart A, Martin AR (1967): An analysis of facilitation of transmitter release at the neuromuscular junction of the frog. J Physiol 193: 679-694.
Noda M, Shimizu S, Tanabe T, Takai T, Kayano T, Ikeda T, Minamino N (1983): Primary structure of Electrophorus electricus sodium channel deduced from cDNA sequence. $\mathrm{Na}$ ture 312:121-127.

- Pedersen KK, Nielsen OB, Overgaard K (2013): Effects of high-frequency stimulation and doublets on dynamic contractions in rat soleus muscle exposed to normal and high extracellular $\left[\mathrm{K}^{+}\right]$. Physiol Rep 1:e00026.

Rack PM, Westbury DR (1969): The effects of length and stimulus rate on tension in the isometric cat soleus muscle. J Physiol 204:443.

Rome LC (2006): Design and function of superfast muscles: new insights into the physiology of skeletal muscle. Annu Rev Physiol 68:193221.

Rome LC, Cook C, Syme DA, Connaughton MA, Ashley-Ross M, Klimov A, Goldman YE (1999): Trading force for speed: why superfast crossbridge kinetics leads to superlow forces. Proc Natl Acad Sci USA 96:5826-5831.

Rome LC, Syme DA, Hollingworth S, Lindstedt SL, Baylor SM (1996): The whistle and the rattle: the design of sound producing muscles. Proc Natl Acad Sci USA 93:8095-8100.

-Sweeney JD (2009): Transcutaneous muscle stimulation. In: TASER ${ }^{\circledR}$ Conducted Electrical Weapons: Physiology, Pathology, and Law (pp. 51-62). Springer USA.

- Thomas CK, Johansson RS, Bigland-Ritchie B (1999): Pattern of pulses that maximize force output from single human thenar motor units. J Neurophysiol 82:3188-3195.
Volta A (1800): On the electricity excited by the mere contact of conducting substances of different kinds (letter from Mr. Alexander Volta, FRS Professor of Natural Philosophy in the University of Pavia, to the Rt. Hon. Sir Joseph Banks, Bart. KBPRS). Philos Trans R Soc Lond 90:403-431.

von Humboldt A (1807): Jagd und Kampf der elektrischen Aale mit Pferden. Aus den Reiseberichten des Hrn. Freiherrn Alexander v. Humboldt. Ann Phys 25:34-43.

Westby GM (1988): The ecology, discharge diversity and predatory behaviour of gymnotiforme electric fish in the coastal streams of French Guiana. Behav Ecol Sociobiol 22:341354.

Williamson H, Walsh J (1775): Experiments and observations on the Gymnotus electricus, or electrical eel. Philos Trans 65:94-101.

Zajac FE, Young JL (1980a): Properties of stimulus trains producing maximum tension-time area per pulse from single motor units in medial gastrocnemius muscle of the cat. J Neurophysiol 43:1206-1220.

Zajac FE, Young JL (1980b): Discharge properties of hindlimb motoneurons in decerebrate cats during locomotion induced by mesencephalic stimulation. J Neurophysiol 43:1-1235. 